\title{
The second Maxwell's relativistic equations of a rapidaly rotating neutron star, based on ZAMO framework (zero angular momentum observers)
}

\author{
Atsnaita Yasrina, Nugroho Adi Pramono, Eny Latifah, Hari Wisodo \\ Jurusan Fisika, FMIPA, Universitas Negeri Malang, Jl. Semarang No. 5, Malang \\ Email atsnaita.yasrina.fmipa@um.ac.id
}

Received 6 October 2016, Revised 20 November 2016, Accepted 12 December 2016

\begin{abstract}
The second Maxwell's relativistic equations of a rapidaly rotating neutron star, based on ZAMO framework (Zero Angular Momentum Observers) has been formulated. The formulations obtained were epresented by differential equations in the radial, polar, and azimuthal components. The ZAMO basis is implemented because the neutron star reviewed as a rotating star. The second Maxwell's equation is important to use as one of the fundamental equations to formulate relativistic magnetic fields dynamics of the neutron stars that rotate rapidly.
\end{abstract}

Keywords: The second Maxwell's equation; neutron star; fast rotation; ZAMO.

\section{Introduction}

Star birth to death through a phase or a so called dynamic star. The dynamics of star depends on the initial mass of the star. Stars that have greater mass will have a shorter life span. This situation is due to the larger mass stars spent fuel faster. The fuel in question is an abundance of nuclei inside stars. While the use of fuel or power generation process at the stars using the fusion reaction. Results of the fusion reaction are the abundance of nuclei.

Stars with masses larger can perform the fusion reaction to produce an abundance of nuclei ${ }^{12} \mathrm{C},{ }^{20} \mathrm{Ne},{ }^{16} \mathrm{O}$, even to ${ }^{56} \mathrm{Fe}$. An abundance of heavier nuclei are in the star's core, while others are sequential in the skin surrounding the star's core. Currently star is not able to perform a fusion reaction, and then the thermal pressure is not enough to offset the force of gravity. When a star's core is not able to perform fusion reactions in their cores, then the thermal stress generated is not enough to offset the force of gravity. Therefore the star undergoes gravitational collapse. This situation causes the stars to be very tight for a very short time interval. As a result of this situation is a stellar explosion called a supernova? In the process of supernova, the majority of which contain an abundance of stellar mass atomic nuclei thrown into interstellar space. While a star's core will be the object or compact objects. While a star's core will be the object or compact objects. 
In astronomy that includes a compact object are a white dwarf, neutron stars, and black holes. For stars with high mass categories, then after a phase of supernova, the core of the star becomes a neutron star or a black hole. The star's core is still experiencing gravitational collapse. If the mass of a stellar core collapse gravity less than the mass Oppenheimer-Volkoff $\left(\approx 2-3 M_{\odot}\right)$, then the star into a neutron star (Potekhin, 2011). While, if the mass of a star's core exceeds the Oppenheimer-Volkoff mass, the gravitational collapse can not be stopped, and the star becomes a black hole.

Stars with masses $\gtrsim 6-8 M_{\odot}$ the end of its dynamic is a neutron star (Camenzind, 2007). The neutron star has mass characteristics $M_{*} \sim 1-2 M_{\odot}$ and has a radius $R_{*} \approx$ $10-14 \mathrm{~km}$. A neutron star can have a maximum mass of $M_{*} \sim 1,5 M_{\odot}$. As for the maximum radius of a neutron star is equal to $R_{*} \sim 3 \mathrm{~km}$ (Shaphiro et al., 2004). Therefore, it has amounted to $\rho \simeq(2-3) \rho_{o}$, with $\rho_{o}=2,8 \times 10^{14} \mathrm{~g} \mathrm{~cm}^{-3}$ is the normal density core (Haensel et al., 2007). While the neutron star gravitational force is $E_{\text {grav }} \sim G M^{2} / R \sim 5 \times 10^{53} \mathrm{erg} \sim 0,2 M c^{2}$, and gravity on the surface of the neutron star is $g \sim G M / R^{2} \sim 2 \times 10^{14} \mathrm{~cm} \mathrm{~s}^{-2}\left(2 \times 10^{11}\right.$ times that of Earth) (Hansel et al., 2007).

The value of the mass density in the neutron star's gravitational force is not the only basic neutron star is part of the compact objects. However compactness of neutron stars are also measured on parameters of compactness is

where

$$
x_{g}=r_{g} / R,
$$

$$
r_{g}=2 G M / c^{2} \approx 2,95 M_{*} / M_{\odot} .
$$

The neutron star has parameters of compactness $x_{g} \sim 0,2-0,4$. The amount parameter compactness of the neutron star, the neutron star is reviewed with general relativity. While many heavenly bodies have a compactness factor much smaller or $x_{g} \ll 1$, which do not require a review of general relativity. For example Sun with compactness parameter of $x_{g} \sim 10^{-6}$.

Another very important characteristic in a neutron star is the magnetic field that is owned neutron star. Neutron star's magnetic field can reach $10^{18} \mathrm{G}$ (Potekhin, 2011). But the magnitude of the magnetic field in a neutron star can be decreased. For example, neutron stars that originally had a magnetic field of $\sim 10^{12} \mathrm{G}$ within $5 \times 10^{6}$ years the magnitude of the magnetic field becomes $\sim 10^{8} \mathrm{G}$ (Zhang, 2007).

A neutron star is detected decreasing magnetic field is found in the dual system (Batacharya, 2002). Meanwhile, if the neutron stars are in multiple systems, then the neutron star's accretion process with a companion stars. Therefore many astrophysicists who found a decrease in the magnetic field in a neutron star neutron star would make the process of accretion, (Anzer et al., 1979; Choudhuri et al., 2002; Cumming et al., 2001; Ho, 2011; Konar et al., 1996; Lovelace et al., 2005; Melatos et al., 2001).

The theoretical study related to a decrease in the magnetic field of which the formulation of equations reduced magnetic field. Equation reduction in the neutron star's magnetic field has been studied. This study reviewed neutron stars do accretion. However, this study within the framework of non-relativistic and non-rotating neutron stars. The resulting equation shows the dynamics of the magnetic field associated with 
the accretion rate (Cumming, et al., 2001). Studies in relativistic been studied by generating an electromagnetic field which is stationary in space time Schwarzschild (Anderson, et al., 1970). The electromagnetic field is needed to assess the dynamics of the magnetic field. But the electric field in the Schwarzschild background neutron star results is not the solution of the equation Maxwell (Sengupta, 1995). In 1997 Sengupta explained ohmic decay rate in the space of time Schwarzschild. While decay ohmic one that is considered responsible for causing the magnitude of the magnetic field in a neutron star. Effects compact neutron star affects the curvature of space and time. Time decline in the magnetic field is shorter if examined on a curved space-time rather than space-time flat (Geppert, et al., 2000). Spinning neutron star and studied relativistic Muslinov and Tsygan in his article in 1992.

In a study about the decline in the magnetic field required magnetic field dynamics equations can be obtained from the relativistic Maxwell equations. Maxwell equations have been formulated solutions in the space of time slowly rotating neutron star with a neutron star around there is no material or neutron star did accretion, with the electromagnetic field slowly rotating neutron star is measured by an observer ZAMO (Zero Angular Momentum Observers) (Rezzolla et al, 2004), Study of rotating star or star rotates using metrics to require a review by ZAMO (Rezzolla et al, 2004a) (Camenzind, 2007). Has generated magnetic field dynamics equations for slowly rotating neutron stars and there is the matter around the neutron star (Yasrina, et al., 2014). The first equation in the relativistic Maxwell spinning neutron star slowly and do accretion has been obtained to be used to formulate the dynamics equations in the neutron star's magnetic field (Yasrina, 2016).

\section{Research Methods}

This research is a theoretical-mathematical study analytically. Therefore, this research method is a literature review. Research procedures to obtain the second equation formulation of the relativistic Maxwell rapidly spinning neutron star ZAMO based framework (shown in Figure (1).

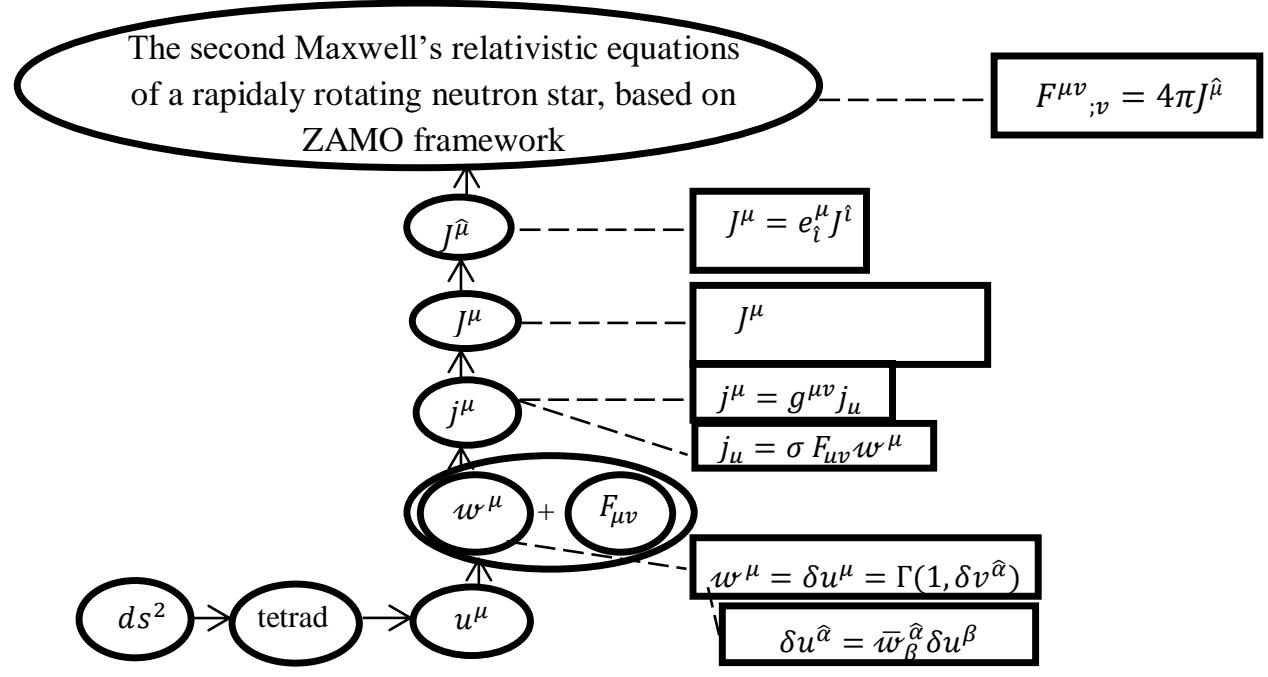

Figure 1. Research procedures to obtain the formulation of four current density 


\section{Results and Discussion}

In a coordinate system $(c t, r, \theta, \varphi)$, the rapidly rotation metric for a rotating relativistic stars is

$$
d s^{2}=-e^{2 \phi} d t^{2}+e^{2 \lambda} r^{2} \sin ^{2} \theta(d \varphi-\omega d t)^{2}+e^{2 \alpha}\left(d r^{2}+r^{2} d \theta^{2}\right)
$$

where $\omega(r)$ can be interpreted as the angular velocityof a free falling (inertia) frame and $\phi, \lambda, \omega, \alpha$ are function that depends on $r$ dan $\theta$ (Gregory,et al., 1994). Equation (3) shows that the component matrix rapidly rotating neutron star is

$$
g_{\mu \nu}=\left[\begin{array}{cccc}
-\left(e^{2 \phi}-e^{2 \lambda} r^{2} \sin ^{2} \theta \omega^{2}\right) & 0 & 0 & -e^{2 \lambda} r^{2} \sin ^{2} \theta \omega \\
0 & e^{2 \alpha} & 0 & 0 \\
0 & 0 & e^{2 \alpha} r^{2} & 0 \\
-e^{2 \lambda} r^{2} \sin ^{2} \theta \omega & 0 & 0 & e^{2 \lambda} r^{2} \sin ^{2} \theta
\end{array}\right] .
$$

The componets of tetrad $\left\{e_{\widehat{\mu}}\right\}=\left(e_{\widehat{0}}, e_{\hat{r}}, e_{\widehat{\theta}}, e_{\widehat{\varphi}}\right)$ carried by ZAMO observer are

$$
\begin{gathered}
e_{\widehat{0}}^{v}=e^{-\phi}(1,0,0, \omega), \\
e_{\hat{r}}^{v}=e^{-\alpha}(0,1,0,0), \\
e_{\widehat{\theta}}^{v}=e^{-\alpha} r^{-1}(0,0,1,0), \\
e_{\widehat{\varphi}}^{v}=\frac{-e^{2 \phi}+e^{2 \lambda} r^{2} \sin ^{2} \theta \omega^{2}}{e^{\phi} e^{\lambda} r \sin \theta} e^{-\phi}(0,0,0,1),
\end{gathered}
$$

and the 1 -form $\left\{w^{\widehat{\mu}}\right\}=\left(w^{\widehat{0}}, w^{\hat{r}}, w^{\widehat{\theta}}, w^{\widehat{\varphi}}\right)$ are

$$
\begin{gathered}
w_{v}^{\widehat{0}}=\left(e^{2 \phi}-e^{2 \lambda} r^{2} \sin ^{2} \theta \omega^{2}\right)^{\frac{1}{2}}(1,0,0,0), w_{v}^{\hat{r}}=e^{\alpha}(0,1,0,0), \\
w_{v}^{\widehat{\theta}}=e^{\alpha} r(0,0,1,0), \\
w_{v}^{\widehat{\varphi}}=e^{\lambda} r \sin \theta(-\omega, 0,0,1)
\end{gathered}
$$

(Yasrina, 2015a). Four-velocity componets for metric in equation (3) are

where

$$
\begin{aligned}
u^{\mu} & =\frac{e^{-\phi}}{\sqrt{1-V^{2}}}(1,0,0, \omega) ; \\
u_{\mu} & =-\frac{e^{\phi}}{\sqrt{1-V^{2}}}(1,0,0,0),
\end{aligned}
$$

$$
V^{2}=e^{2 \phi}\left[e^{2 \lambda} r^{2} \sin ^{2} \theta(\Omega-\omega)^{2}+e^{2 \alpha}\left(v^{r}\right)^{2}+e^{2 \alpha} r^{2}\left(v^{\theta}\right)^{2}\right]
$$

(Yasrina, 2015a). While the conductor four-velocity can be obtained from the equation (12) that is

$\delta u^{\mu}=\Gamma\left(1, \delta v^{\widehat{\alpha}}\right)=\Gamma\left(1, e^{-\alpha} \delta v^{\hat{r}}, e^{-\alpha} r^{-1} \delta v^{\widehat{\theta}}, \frac{\left(-e^{2 \phi}+e^{2 \lambda} r^{2} \omega^{2} \sin ^{2} \theta\right)^{\frac{1}{2}}}{e^{\phi} e^{\lambda} r \sin \theta} \delta v^{\widehat{\varphi}}\right)=w^{\alpha}$,

with $\Gamma$ is

$$
\Gamma=\left[-g_{00}\left(1+\frac{g_{\alpha \beta}\left(\delta v^{\alpha} \delta v^{\beta}\right)}{g_{00}}\right)\right]^{-\frac{1}{2}}
$$


(Rezzolla dkk, 2004b). In this case, it is assumed small velocity pertubation for which $\delta v^{\alpha} \ll 1$, so the equation (10) into

$$
\Gamma \approx\left[-g_{00}\right]^{-\frac{1}{2}} \approx\left(e^{2 \phi}-e^{2 \lambda} r^{2} \omega^{2} \sin ^{2} \theta\right)^{-\frac{1}{2}} .
$$

The general form second pair of Maxwell equation is given by

$$
F_{; v}^{\mu v}=4 \pi J^{\mu} \text {. }
$$

The four-current $J^{\alpha}$ is a sum of convection and $\left(\rho_{e} w^{\alpha}\right)$ conduction currents $\left(j^{\alpha}\right)$

$$
J^{\mu}=\rho_{e} w^{\mu}+j^{\mu} ; j^{\mu} w_{\mu} \equiv 0,
$$

with $w$ being the conductor four-velocity, $\rho_{e}$ the proper charge density, dan $\left(j^{\mu}\right)$ counduction curret. The conduction curret is

$$
j_{\mu}=\sigma F_{\mu v} w^{\mu}
$$

with $\sigma$ is electrial conductivity. The equation (14) requires the covariance tensor electromagnetic field that can be obtained from the metric in equation (3). Hence the conduction current covariance component is

$$
\begin{aligned}
& j_{0}=\frac{\sigma}{\sqrt{1-V^{2}}} {\left[-\left(e^{\phi} e^{\alpha} E^{\hat{r}}-e^{\alpha} e^{\lambda} r^{2} \omega \sin \theta B^{\widehat{\theta}}\right) \Gamma e^{-\alpha} \delta v^{\hat{r}}\right.} \\
&+\left(-e^{\phi} E^{\widehat{\theta}}+e^{\alpha} e^{\lambda} r^{2} \omega \sin \theta B^{\hat{r}}\right) \Gamma e^{-\alpha} r^{-1} \delta v^{\widehat{\theta}} \\
&\left.-\left(-e^{2 \phi}+e^{2 \lambda} r^{2} \omega^{2} \sin ^{2} \theta\right) E^{\widehat{\theta}} \Gamma e^{-\phi} \delta v^{\widehat{\varphi}}\right] \\
& j_{r}=\frac{\sigma}{\sqrt{1-V^{2}}}[\left(e^{\phi} e^{\alpha} E^{\hat{r}}-e^{\alpha} e^{\lambda} r^{2} \omega \sin \theta B^{\widehat{\theta}}\right) \Gamma \\
&-\left(-e^{2 \phi}+e^{2 \lambda} r^{2} \omega^{2} \sin ^{2} \theta\right)^{\frac{1}{2}} B^{\widehat{\varphi}} \Gamma e^{\alpha} e^{\phi} r^{-1} \delta v^{\widehat{\theta}} \\
&\left.-\left(-e^{2 \phi}+e^{2 \lambda} r^{2} \omega^{2} \sin ^{2} \theta\right)^{\frac{1}{2}} B^{\widehat{\theta}} \Gamma e^{\alpha} e^{\phi} \delta v^{\widehat{\varphi}}\right] \\
& j_{\theta}=\frac{\sigma}{\sqrt{1-V^{2}}} {\left[-\left(e^{\phi} E^{\widehat{\theta}}+e^{\alpha} e^{\lambda} r^{2} \omega \sin ^{\hat{r}}\right) \Gamma\right.} \\
&+\left(-e^{2 \phi}+e^{2 \lambda} r^{2} \omega^{2} \sin ^{2} \theta\right)^{\frac{1}{2}} B^{\widehat{\varphi}} \Gamma e^{\alpha} e^{-\phi} \delta v^{\hat{r}} \\
&\left.-\left(-e^{2 \phi}+e^{2 \lambda} r^{2} \omega^{2} \sin ^{2} \theta\right)^{\frac{1}{2}} B^{\hat{r}} \Gamma e^{\alpha} e^{-\phi} r \delta v^{\widehat{\varphi}}\right], \\
& j_{\varphi}=\frac{\sigma}{\sqrt{1-V^{2}}}\left[\left(-e^{2 \phi}+e^{2 \lambda} r^{2} \omega^{2} \sin ^{2} \theta\right) E^{\widehat{\theta}} \Gamma e^{\lambda} r \sin \theta+e^{\lambda} r \sin ^{\widehat{\theta}} \Gamma B^{\widehat{\theta}} \delta v^{\hat{r}}+e^{\lambda} r \sin \theta B^{\widehat{\theta}} \Gamma \delta v^{\widehat{\theta}}\right] .
\end{aligned}
$$

While the four-current in ZAMO framework for rapidly rotating neutron star is

$$
\begin{gathered}
J^{\widehat{0}}=e^{\phi} \rho_{e} \Gamma-e^{-\phi}\left(j_{0}-\omega j_{\varphi}\right), \\
J^{\hat{r}}=\rho_{e} \Gamma \delta v^{\hat{r}}+e^{-\alpha} j_{r}, \\
J^{\widehat{\theta}}=\rho_{e} \Gamma \delta v^{\widehat{\theta}}+e^{-\alpha} r^{-1} j_{\theta}, \\
J^{\widehat{\varphi}}=\rho_{e} \Gamma \delta v^{\widehat{\theta}}-e^{-2 \phi} \omega j_{o} \frac{\left(e^{\phi} e^{\lambda} r \sin \theta\right)}{\left(-e^{2 \phi}+e^{2 \lambda} r^{2} \omega^{2} \sin ^{2} \theta\right)^{\frac{1}{2}}}+\frac{\left(-e^{2 \phi}+e^{2 \lambda} r^{2} \omega^{2} \sin ^{2} \theta\right)^{\frac{1}{2}}}{e^{\phi} e^{\lambda} r \sin \theta} j_{\varphi},
\end{gathered}
$$

with $j_{0}, j_{r}, j_{\theta}$, and $j_{\varphi}$ is expressed in the equation (15). The equations (9), (11), (15), and (16) was presented at Seminar Nasional Fisika dan Pembelajarannya 2016 in Universitas Negeri Malang. 
The contavarian electromagnetic tensor rapidly rotating neutron star within the ZAMO framework observers is

$$
\begin{aligned}
& F^{00}=F^{11}=F^{22}=F^{33}=0, \\
& F^{01}=-F^{10}=\left(-Y^{-1} e^{-\alpha} B^{r}\right)=-e^{-\phi-\alpha} \sqrt{1-V^{2}} B^{\hat{r}}, \\
& F^{02}=-F^{20}=\left(Y^{-1} B^{\theta}\right)=e^{-\phi-\alpha} r^{-1} \sqrt{1-V^{2}} B^{\widehat{\theta}} \\
& F^{03}=-F^{30}=-\left(Y^{-1} B^{\varphi}\right)=\frac{\left(-e^{2 \phi}+e^{2 \lambda} r^{2} \sin ^{2} \theta \omega^{2}\right)^{1 / 2}}{e^{2 \phi+\lambda} r \sin \theta} \sqrt{1-V^{2}} B^{\widehat{\varphi}}, \\
& F^{12}=-F^{21}=-\left(X^{-1} E_{\varphi}\right)=-\frac{\left(-e^{2 \phi}+e^{2 \lambda} r^{2} \sin ^{2} \theta \omega^{2}\right)^{\frac{1}{2}}}{e^{2 \alpha+\phi} r} \sqrt{1-V^{2}} E^{\widehat{\varphi}}, \\
& F^{13}=-F^{31}=-\left(X^{-1} E_{\theta}-Y^{-1} \omega B^{r}\right)=-\left[\frac{E^{\widehat{\theta}}}{e^{\alpha+\lambda} r \sin \theta}-\frac{\omega}{e^{\phi+\alpha}} B^{\hat{r}}\right] \sqrt{1-V^{2}}, \\
& F^{23}=-F^{32}=-\left(X^{-1} E_{r}-Y^{-1} \omega B^{\theta}\right)=-\left[\frac{E^{\widehat{r}}}{e^{\alpha+\lambda} r^{2} \sin \theta}-\frac{\omega}{e^{\phi+\alpha}} B^{\widehat{\theta}}\right] \sqrt{1-V^{2}}
\end{aligned}
$$

(Yasrina, 2015b). The general form second pair of Maxwell equation can be obtained by substituting equation (16) and (17) to the equation (12), with $J^{\widehat{\mu}}$ also within the ZAMO framework, in order to obtain

$$
\begin{aligned}
& -\left(e^{\alpha+\lambda} r^{2} \sin \theta \sqrt{1-V^{2}} B^{\hat{r}}\right)_{, r}+\left(\left(e^{\phi} e^{2 \alpha} e^{\lambda} r^{2} \sin \theta\right) e^{\alpha+\lambda} r \sin \theta \sqrt{1-V^{2}} B^{\widehat{\theta}}\right)_{, \theta} \\
& +\left(e^{-\phi+2 \alpha} r\left(-e^{2 \phi}+e^{2 \lambda} r^{2} \sin ^{2} \theta \omega^{2}\right)^{1 / 2} \sqrt{1-V^{2}} B^{\widehat{\varphi}}\right)_{, \varphi}=\left(e^{\phi} e^{2 \alpha} e^{\lambda} r^{2} \sin \theta\right) 4 \pi J^{\widehat{0}} \\
& \left(e^{\alpha+\lambda} r^{2} \sin \theta \sqrt{1-V^{2}} B^{\hat{r}}\right)_{, t}-\left(e^{\lambda} r \sin \theta\left(-e^{2 \phi}+e^{2 \lambda} r^{2} \sin ^{2} \theta \omega^{2}\right)^{\frac{1}{2}} \sqrt{1-V^{2}} E^{\widehat{\varphi}}\right)_{, r} \\
& -\left(\left(e^{\phi} e^{2 \alpha} e^{\lambda} r^{2} \sin \theta\right)\left[\frac{E^{\widehat{\theta}}}{e^{\alpha+\lambda} r \sin \theta}-\frac{\omega}{e^{\phi+\alpha}} B^{\hat{r}}\right] \sqrt{1-V^{2}}\right)_{, \varphi}=\left(e^{\phi} e^{2 \alpha} e^{\lambda} r^{2} \sin \theta\right) 4 \pi J^{\hat{r}},
\end{aligned}
$$

$$
\begin{aligned}
& -\left(e^{\alpha+\lambda} r \sin \theta \sqrt{1-V^{2}} B^{\widehat{\theta}}\right)_{, t}+\left(e^{\alpha+\lambda} r \sin \theta\left(-e^{2 \phi}+e^{2 \lambda} r^{2} \sin ^{2} \theta \omega^{2}\right)^{\frac{1}{2}} \sqrt{1-V^{2}} E^{\widehat{\varphi}}\right)_{, r} \\
& -\left(\left(e^{\phi} e^{2 \alpha} e^{\lambda} r^{2} \sin \theta\right)\left[\frac{E^{\widehat{r}}}{e^{\alpha+\lambda} r^{2} \sin \theta}-\frac{\omega}{e^{\phi+\alpha_{r}}} B^{\widehat{\theta}}\right] \sqrt{1-V^{2}}\right)_{, 3}=\left(e^{\phi} e^{2 \alpha} e^{\lambda} r^{2} \sin \theta\right) 4 \pi J^{\widehat{\theta}},
\end{aligned}
$$

$$
\begin{aligned}
& \left(e^{-\phi+2 \alpha} r\left(-e^{2 \phi}+e^{2 \lambda} r^{2} \sin ^{2} \theta \omega^{2}\right)^{\frac{1}{2}} \sqrt{1-V^{2}} B^{\widehat{\varphi}}\right)_{, t} \\
& +\left(\left(e^{\phi} e^{2 \alpha} e^{\lambda} r^{2} \sin \theta\right)\left[\frac{E^{\widehat{\theta}}}{e^{\alpha+\lambda} r \sin \theta}-\frac{\omega}{e^{\phi+\alpha}} B^{\hat{r}}\right] \sqrt{1-V^{2}}\right)_{, r} \\
& +\left(\left(e^{\phi} e^{2 \alpha} e^{\lambda} r^{2} \sin \theta\right)\left[\frac{E^{\widehat{r}}}{e^{\alpha+\lambda} r^{2} \sin \theta}-\frac{\omega}{e^{\phi+\alpha_{r}}} B^{\widehat{\theta}}\right] \sqrt{1-V^{2}}\right)_{, \theta}= \\
& \left(e^{\phi} e^{2 \alpha} e^{\lambda} r^{2} \sin \theta\right) 4 \pi J^{\widehat{\varphi}} .
\end{aligned}
$$

Equation (18) to (21) and the first equation Maxwell equations can be used to formulate the dynamics of the magnetic field in rapidly rotating neutron star that does accretion. 


\section{Conclusion}

The equation (18) to (21) are the general form second pair of Maxwell equation in in rapidly rotating neutron star that does accretion within the ZAMO framework. The general form second pair of Maxwell equation obtained are represented by differential equations in the radial, polar, and azimuthal components. The second Maxwell's equation is important to use as one of the fundamental equations to formulate relativistic magnetic fields dynamics of the neutron stars that rotate rapidly.

\section{References}

Anderson, J. L., Cohen, J.M., (1970), Gravitational Collapse of Magnetic Neutron Stars. Astropys. Space Science. 9, 146.

Anzer, U., Börner, G., (1979), Accretion by Neutron Stars: Accretion Disk and Rotating Magnetic Field, Astron. Astrophysics, 133-139

Bhattacharya, D., (2002). Evolution of Neutron Stars Magnetic Fields. Jurnal Astrophysics, The Astrophysical Jurnal. Astr, 67-72.

Camenzind, M., (2007), Compact Objects in Astrophysics White Dwarfs, Neutron Stars, and Black Hole., Verlag Berlin Heidelberg: Springer.

Choundhuri A. R., Konar S., (2002), Diamagnetic Screening of the Magnetic Field in Accreting Neuyron stars, http://www.arXiv.astro-ph/0108229, 27 January 2002

Cumming, A., Zweibel, E., Bildsten, L., (2001), Magnetic Screening in Accreting Neutron Stars, http://www.arXiv.astro-ph/0102178, 9 February 2001

Geppert, U., Page, D., Zannias, T., (2000.), General Relativitic Treatment Of The Thermal, Magnetic, And Rotational Evolution of Isolated Neutron Stars With Crustal Magnetic Fields. Astron-Astrophys. 2-1066.

Gregory, B., Cook, Stuart,L., Shapiro, Saul, A., Teukolsky, (1994), Rapidly Rotating Neutron Stars in General Relativity: Realistic Equations of State, The American Astronomical Society: Astrophysical Journal, 424: 828-845.

Haensel, P., Potekhin, A.Y., Yakovlev, D.G., (2007), Neutron Stars 1 Equation of State and Structure. New York: Springer.

Ho,Wynn. C. G., (2011), Evolution of a Burried Magnetic Field in The Central Compact Object Neutron Stars, http://www.arXiv.astro-ph 11024870v1, 23 February 2011

Konar, S., Bhattacharya, D., (1996), Magnetic Field Evolution of Accreting Neutron Stars, R. Astron. Soc. 284, 311-317

Lovelace R. V. E., Romanova M. M., (2005), Sreening of The Magnetic Field of Disk Accreting Stars, The Astrophysical Jurnal, 625:957-965

Melatos A., dkk, (2001), Hydromagnetic Structure of a Neutron Stars Accreting at Its Polar Caps, Astron Soc Aust 421-430

Muslinov, A., Tsygan, A.I., (1992), General Relativistic Electric Potential Drops Above Pulsar Polar Caps, MNRAS. 255, 61.

Potekhin, A.Y., (2011). The Physics Of Neutron Stars. astro-ph. SR, 1235-1256. 
Rezzolla L., dkk, (2004),. General Relativitic Elektromagnetic Fields of a Slowly Rotating Magnetized Neutron Stars I. Formulation of the Equation. MNRAS. 119.

Rezzola L., dkk., (2004b). Electromagnetic Fields in the Exterior of an Oscillating Relativistic Sta-I. General Expressions and Application to A Rotating Magnetic Dipole. MNAR. 1-21

Sengupta, S., (1995), General Relativistic Effect on The Induced Electric Field Exterior To Pulsar. ApJ. 449, 224.

Sengupta, S., (1997), General Relativistic Effect on The Ohmic Decay Of Crustal Magnetic Fields in Neutron Stars, ApJ, 479, L133

Shaphiro, L. S., Teukolsky, S. A., (2004), Black Hole, White Dwarfs, and Neutron stars, Verlag: Wiley-VCH

Yasrina, A., Rosyid, M.F., (2013). Tentang Medan Elektromegnetik Relativistik di Bintang Neutron yang Berotasi Lambat. Tesis tidak diterbitkan. Jurusan Fisika Universitas Gadjah Mada

Yasrina, A., (2015a). Tensor Elektromagnetik Bintang Neutron yang Berotasi Cepat Diukur oleh Pengamat ZAMO (Zero Angular Momentum Observers), ISBN 978-602-71273-1-9, A-1-A-5.

Yasrina, A., (2015b), Tensor Kontravarian Medan Elektromagnetik Bintang Neutron yang Berotasi Cepat Diukur oleh Pengamat ZAMO (Zero Angular Momentum Observers), Jurnal Fisika, 5: 41-46

Yasrina, A., (2016), Persamaan Pertama Maxwell Relativistik Bintang Neutron yang Berotasi Cepat Diukur oleh Pengamat ZAMO (Zero Angular Momentum Observers), Journals of Scientect, Vol. 2 (1): 144-150

Zhang, C. M., (1997), Accretion Induced Crust Screening For The Magnetic Filed Decay of Neutron Stars, Astron. Astropys, 1995-200 